\title{
LARYNX, Etc.
}

Mouret and Denoyès.-On the Employment of High-Frequency Curients in Oto-Laryngology. " Revue Hebdom. de Laryng., etc.," May 26, 1902.

After giving a short description of the technique of this treatment, the authors report the following cases.

1. Paralysis of Right Recurrent Laryngeal Nerve, and Enlargement of Right Lobe of Thyroid Gland. - The goitre had first been noted about four years ago, and had gradually increased. In March, 1898, patient caught a cold and became aphonic, but did not consult a doctor till July, 1899. She was then treated for ten months with sprays and painting the throat with zinc chloride, and for one month with faradization, but without obtaining any improvement. She consulted Mouret in October, 1900. Her voice was then very low, the right rocal cord was in the cadaveric position, and a goitre existed on the right side of the neck. No change was noted in the mucous membrane of larynx, etc. A course of iodide of potassium produced no effect. Six applications of a galvanic current to the goitre gave rise to tachycardia and palpitations. Galvanism, therefore, was stopped, and highfrequency currents applied instead. This treatment was carried out, with interruptions, from December to July. The patient's voice had improved, but the right vocal cord remained fixed in the cadaveric position.

2. Paralysis of Iight Recurrent Nerve following a Cold.-Treatment by sprays of benzoate of soda, iodine, externally over larynx, etc., carried on for a month, reduced the state of congestion of the parts, but the right cord remained fixed in the cadaveric position. During a second month the larynx was painted with zinc chloride, and both galvanic and faradic currents applied, with the result that the adductor muscles of the right cord regained their power, whilst the abductors remained quite paralytic. By the end of another month indications of very slight return of power in the abductors were present, but the patient complained that the treatment exhausted him. Treatment by high-frequency currents was then begun. There was almost immediate improvement in the subjective sensations and in the power of the voice. After eighteen applications the right cord moved as freely as the left; recovery was complete, and there had been no relapse when patient was last seen-i.e., after ten months.

The authors have also applied high-frequency currents to cases of deafness and tinnitus due to sclerosing otitis. In all their cases the noted an immediate improvement in the tinnitus after each sitting. The improvement was at first of very short duration, but gradually lasted longer, but in only one case did they obtain a cure. Deafness was not benefited. Arthur J. Hutchison.

\section{THYROID AND TRACHEA.}

von Shroetter, Herman.-Tracheal Scleroma. "Journal of Ophthal., Otol., and Laryn.," March, 1902.

A woman, aged twenty-eight, born in Hungary, but having lived for' the past fifteen years in Styria, presented herself in December 15, 189\%, suffering with cough, severe dyspncea, and aphonia. Her family history 
contained no specific taint. One brother had suffered from severe dyspnœa with stridulous breathing for four or five years, and another member of the family was similarly affected.

On admission the patient, who was of robust appearance, was labouring with severe stridulous dyspncea, pulse 72, respiration 20 . During inspiration the larynx was retracted, the veins of the neck were distended. The cervical lymphatics were not enlarged.

Laryngoscopy revealed nothing abnormal about the vocal cords or parts above their level, but under the cords were seen projecting red excrescences superficially striated and symmetrically distributed, which on probing were soft.

In the trachea on a level with the fifth ring was a swelling the shape of a button, originating from the left wall, the base of which was formed by a red swelling sagittally placed. The cone, which much obstructed the orifice, was the colour of yellow soap with a slimy surface. Over the tumour in the front part of the trachea was a grayish-white cicatrix with sharp edges, hiding the view.

A diagnosis of tracheal scleroma was made, and subsequently was confirmed by bacteriological examination.

Treatment.-Inhalations and morphine were tried, but, giving no relief, operative measures were decided upon.

December 20: After cocainization, the most obstructive portions of the growth were removed by suitably-curved forceps and curettage. Hiemorrhage was but slight and the relief afforded to patient great. The next day more tissue was removed.

December 20: No fever nor stridor, general condition good. Trachea was dilated with a medium-sized English catheter.

December 23: A No. 3 hard rubber sound was comfortably passed; in the evening there was soreness and burning pain in trachea.

December 24 : There was redness of the posterior extremity of right rocal cord, but prominences under the latter were much less than two days ago. Dilatation was suspended and ice applied. Respiration free.

December 25: Alterations in right cord more marked, succulent red tumefaction obvious over its posterior part. A soft sound (No. 24) was introduced for ten minutes; later in the day a dyspncic attack superrened, lasting fifteen minutes; this was relieved by morphine. No fever. Dilatation was persevered with, and by January 9 the swellings under the cord had atrophied. Treatment was continued, and early in February, 1898, her condition had so improved that she was discharged. At this time the mucous membrane at the bifurcation of the trachea was smooth and of normal thickness, but that at the upper part was somewhat thickened, rendering rings of trachea invisible. There was no swelling under the left cord, and only a callous narrow ridge along the edge of the inferior aspect of the right cord. Colour and mobility of cords were good and voice normal.

In November of the same year she returned with a recurrence of the growth. The anterior portion of the vocal cord was hypertrophied, and there was tumefaction extending from the anterior commissure to the tracheal ring ; under the left cord was a prominent swelling.

She was again operated upon. The swelling in the anterior part of the subglottic region was excised, followed by daily dilatation with No. 10 bougies for three weeks, which gave entire relief.

During 1899 she reported herself twice, and although suffering no inconvenience, the excrescences in the subglottic region had re- 
appeared. The same systematic treatment as before was undergone, affording great improvement.

In December, 1900, the patient again returned with dyspnoea, aphonia, and cough, attended with expectoration of reddish-yellow particles. Tubercle bacilli were absent from expectoration, and heart and lungs were sound. Laryngoscopy revealed the inferior border of left ventricle moderately enlarged and red at anterior part, left vocal cord much hypertrophied and roughened, infiltration in anterior part of subglottic space. Structure of left cord was irrecognisable, being replaced by masses of reddish-gray succulent tissue. The infiltration was especially marked in the middle of the glottis, and extended on to the anterior laryngo-tracheal wall down to the upper border of fourth ring of trachea. The vegetations of vocal cord were of soft consistence: those on the anterior wall were far harder. Mobility of the left cord was greatly hampered. Nucous membrane on post-laryngeal wall normal. Opposite the fifth and sixth rings of trachea the infiltration of three years ago was replaced by a slightly thickened membrane: other regions of trachea normal. There was no reappearance of growth in the portions of trachea affected in December, 1897. In two sittings the infiltrations were removed by écraseur and forceps, followed by use of bougies for a week. The patient was again discharged, breathing easily. Left vocal cord normal in every way, voice hoarse. In February, 1901, her condition was excellent, voice clear, and from all points of view was better than in February 28, 1898.

In discussing the treatment, the author is strongly in favour of methodical dilatation, combined with lateral compression, after L. r. Schroetter's method, and deprecates laryngo-fissure, save in cases where endo-laryngeal, endo-tracheal, or bronchoscopic interventions fail, or as a last resort.

As regards medical treatment, iodide of potash, salicylic acid, and injections of sublimate, have proved useless, and the sero-therapy of Pawlowsky is still sub judice.

The writer believes that although scleroma is for the most part endemic and often sporadic, still it may be found in any portion of Europe.

H. Clayton For.

\section{EAR.}

Dunn, John (Richmond, Va.).-The Inferior Occipital Plate of the Mastoid, with especial reference to Bezold's Mastoiditis. "Arch. of Otol.," vol. xxxi., No. 2.

This is the space a sixth to a quarter of an inch wide on the undersurface of the mastoid internal to the digastric groove. It has a portion of the sigmoid sinus just above its inner table, and in some cases it contains air cells. In those cases of Bezold's mastoiditis which do not readily recover after opening the mastoid it is probable that the perforation has taken place through this part of the bone.

Dundas Grant.

Lenoir, Oliver.-The Morphological Value of the Supra-meatal Spine and Tache Spongieuse in Antrectomy. "Annals of Otology, Rhinology, and Laryngology," February, 1902.

The author demurs as to the developmental origin of the spine held by Poirier, who considers it a portion of the tympanic bone, designatin." it the "épine tympanale." 